\title{
Factores asociados a complicaciones crónicas de diabetes mellitus tipo 2 en pacientes de un hospital general del Seguro Social de Salud del Perú
}

\section{Associated factors with chronic complications of type-2-diabetes mellitus among patients from a general hospital of the Peruvian social health insurance}

\author{
Juan Villacorta Santamato, , Nélida Hilario Huapaya ${ }^{2,3, b}$, Jorge Inolopú Cucche ${ }^{2,3, c}$, Leonel Terrel Gutierrez ${ }^{4, \mathrm{~d}}$, Rosa Labán Hijar ${ }^{4, e}$, \\ José Del Aguila ${ }^{1, \mathrm{f}}$, Cesar Ugarte-Gil ${ }^{2,5, g}$, Yamilée Hurtado-Roca ${ }^{6, h}$ \\ ${ }^{1}$ Hospital I Aurelio Díaz Ufano y Peral, EsSalud. Lima, Perú. \\ ${ }^{2}$ Instituto de Medicina Tropical Alexander von Humboldt, Universidad Peruana Cayetano Heredia. Lima, Perú. \\ ${ }^{3}$ Facultad de Salud Pública, Universidad Peruana Cayetano Heredia. Lima, Perú. \\ ${ }^{4}$ Hospital Guillermo Almenara Irigoyen, EsSalud. Lima, Perú. \\ ${ }^{5}$ Facultad de Medicina, Universidad Peruana Cayetano Heredia. Lima, Perú. \\ ${ }^{6}$ Instituto de Evaluación de Tecnologias en Salud e Investigación (IETSI), EsSalud. Lima, Perú. \\ ${ }^{a}$ ORCID: https://orcid.org/0000-0002-4154-3234 \\ ${ }^{b}$ ORCID: https://orcid.org/0000-0001-9664-1803 \\ ${ }^{c}$ ORCID: https://orcid.org/0000-0002-2312-8387 \\ d ORCID: https://orcid.org/0000-0002-1066-3823 \\ ${ }^{e}$ ORCID: https://orcid.org/0000-0001-8339-7514 \\ 'ORCID: https://orcid.org/0000-0003-0606-6775 \\ ${ }^{9}$ ORCID: https://orcid.org/0000-0002-2833-9087

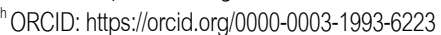

Correspondencia:

Yamilée Hurtado Roca

leda.hurtado@essalud.gob.pe

Recibido: 30 de julio 2020

Aprobado: 4 de septiembre 2020

Publicación en línea: 30 de septiembre 2020

Conflictos de interés: El autor declara no tener conflictos de interés.

Fuente de financiamiento: Instituto de Evaluación de Tecnologías en Salud e Investigación (IETSI), EsSalud.

Citar como: Villacorta J, Hilario N Inolopú J, Terrel L, Labán R, Del Aguila J, et al. Factores asociados a complicaciones crónicas de diabetes mellitus tipo 2 en pacientes de un hospital general del Seguro Social de Salud del Perú. An Fac med. 2020;81(3):308-15. DOl: https://doi. org/10.15381/anales.v81i3.17260.
An Fac med. 2020;81(3):308-15. / DOI: https://doi.org/10.15381/anales.v81i3.17260

Resumen

Introducción. Las complicaciones crónicas por diabetes mellitus tipo 2 (DMT2) elevan la mortalidad y carga de enfermedad en Perú, siendo necesario evaluar su prevalencia y factores asociados. Métodos. Estudio transversal, analítico, en registros de 212 pacientes adultos con DMT2 atendidos por consulta externa en un hospital general del Seguro Social del Perú, durante el año 2017. Resultados. El 71,6\% de los pacientes fueron mayores de 60 años y con un tiempo de enfermedad menor de 6 años (40,5\%). El 38,7\% de los pacientes tuvieron alguna complicación crónica, principalmente nefropatía (48,8\%) y neuropatía (45,1\%). La hipertensión arterial $(52,4 \%)$ y la obesidad $(43,7 \%)$ fueron las comorbilidades más frecuentes en pacientes con complicaciones crónicas. Las complicaciones por DMT2 estuvieron asociadas al número de consultas médicas y monitoreo glicémico. Conclusiones. Existen factores de riesgos asociados a las complicaciones crónicas de DMT2.

Palabras clave: Diabetes Mellitus Tipo 2; Complicaciones de la Diabetes; Seguridad Social; Peru (fuente: DeCS BIREME).

Abstract

Introduction. The chronic complications of type-2-diabetes mellitus (T2DM) increase mortality and burden of disease in Peru, then it is necessary to evaluate its prevalence and associated factors. Methods. Cross-sectional study in records of 212 adult outpatients with T2DM attended by a social security general hospital in Peru, during 2017. Results. $71,6 \%$ of patients were older than 60 years and with a time of illness less than 6 years $(40,5 \%) .38,7 \%$ of the patients had any chronic complication, mainly nephropathy $(48,8 \%)$ and neuropathy $(45,1 \%)$. Hypertension $(52,4 \%)$ and obesity $(43,7 \%)$ were the most frequent comorbidities in outpatients with chronic complications. Complications from DMT2 were associated with the medical consultations and glycemic monitoring. Conclusions. There are risk factors associated to chronic complications of DMT2

Keywords: Diabetes Mellitus, Type 2; Diabetes Complications; Social Security; Peru (source: MeSH NLM). 


\section{INTRODUCCIÓN}

La diabetes mellitus (DM) es una enfermedad metabólica caracterizada por deficiencia en la producción o acción de la insulina que promueve cuadros de hiperglicemia, polidipsia, poliuria, polifagia, pérdida de peso, entre otros ${ }^{(1)}$. La diabetes tipo 2 (DMT2) se caracteriza por una secreción deficiente e insuficiente de la insulina (2), incrementándose el riesgo de su desarrollo con la presencia de factores concomitantes ${ }^{(3)}$. De acuerdo a la Federación Internacional de Diabetes, en el 2017, la DM afectó aproximadamente a $425 \mathrm{mi}-$ llones de personas en el mundo ${ }^{(4)}$, y debido a su constante incremento, se espera que para el año 2045 existan 693 millones de personas viviendo con DM ${ }^{(5)}$. En el Perú, de acuerdo al Instituto Nacional de Estadística e Informática, en el 2018, el 3,6\% de la población de 15 y más años de edad reportó estar diagnosticada con DM por un médico alguna vez en su vida ${ }^{(6)}$.

La DMT2 está vinculada a diversas morbilidades ${ }^{(7-10)}$, pero principalmente a complicaciones crónicas micro y macrovasculares causadas por el inadecuado control glicémico ${ }^{(11)}$. Las complicaciones crónicas son el resultado de la progresión de la DMT2, reducen la calidad de vida de los pacientes e incrementan la carga de enfermedad; teniendo un significativo impacto en la economía de países en desarrollo que cursan una transición epidemiológica y presentan un sistema sanitario poco desarrollado ${ }^{(12,13)}$. Adicionalmente, se ha reportado que las complicaciones crónicas de la DMT2 son las principales causas de mortalidad en estos pacientes ${ }^{(14,15,16)}$.

Existe evidencia reciente que evalúa la prevalencia e incidencia de DMT2 en población general en nuestro país (16); sin embargo, no existe literatura publicada sobre las complicaciones crónicas por DMT2 y los factores asociados a estos. Por lo tanto, el objetivo de nuestro estudio fue determinar las complicaciones de DMT2 en los pacientes que recibieron atención sanitaria en un hospital de nivel primario de una zona urbana y proponer un mejor enfoque de seguimiento y tratamiento.

\section{MÉTODOS}

\section{Diseño del estudio}

Se realizó un estudio transversal, analítico, en pacientes con DMT2 atendidos en los servicios de consulta externa del Hospital I Aurelio Díaz Ufano, durante el año 2017. Durante marzo a junio del 2018, se consultaron los registros clínicos, que incluyeron: (i) historia clínica, (ii) registro del sistema informático, (iii) y ficha de seguimiento por enfermería

\section{Población y muestra}

Se analizaron 240 registros clínicos (muestra), aleatorizados a partir de un total de 1999 registros de pacientes con DMT2 atendidos en los servicios de consulta externa de un hospital de atención primaria de la seguridad social durante el año 2017 (población).

\section{Variables del estudio}

De las historias clínicas, se extrajo información sociodemográfica, tipo de aseguramiento, fecha de afiliación a EsSalud, fecha del primer reporte o diagnóstico de DMT2 y comorbilidades: hipertensión arterial, dislipidemia, anemia, artrosis, infección urinaria y glaucoma.

De los registros del sistema informático se obtuvo el total de consultas médicas, medicación prescrita, resultados de pruebas de laboratorio, fechas de las consultas médicas, fechas de citas para pruebas de laboratorio, fechas de emisión de sus respectivos resultados y el tipo de complicación crónica utilizando el código de clasificación internacional de enfermedades (CIE) versión 10: neuropatía diabética (CIE: E11.4, G59.0, G63.2, G99.0 y G62.9), nefropatía diabética (CIE: E11.2 y N08.3), retinopatía diabética (CIE: H36.0), pie diabético (CIE: L98.4), enfermedad arterial periférica (CIE: E11.5), enfermedad cerebrovascular (CIE: I67.9, 169.3, 169.4, 163.0) y enfermedad isquémica $(125.5,125.9,142.0)$ (13). En el caso de pie diabético, se consideró clasificar como tales a aquellos registros con indicación de pie diabético neuro-isquémico o neuropático y úlcera crónica en el pie, siempre que no haya habido alguna otra causa subyacente. De modo similar, los casos de nefropatía definida por CIE con códigos N18.1, N18.5, N18.9 y N04.0, fueron considerados de origen diabético (nefropatía diabética) siempre que no haya alguna otra causa subyacente. De la ficha de seguimiento por enfermería se extrajo información sobre índice de masa corporal, pruebas de glicemia capilar (hemoglucotest), presión arterial, datos sociodemográficos complementarios al registro de filiación en la historia clínica y antecedentes personales de riesgo como sedentarismo.

\section{Análisis estadístico}

Se realizó un análisis bivariado utilizando pruebas paramétricas y no paramétricas para determinar los factores asociados a complicación crónica por DMT2. Para el análisis multivariado, se desarrolló un modelo de regresión de Poisson con varianza robusta para obtener razones de prevalencia (RP) (15), que incluyó variables según criterio estadístico y epidemiológico. Se crearon intervalos de confianza al 95\% y las hipótesis fueron contrastadas con un nivel de significancia del 5\%. El análisis se realizó con el software Stata 15 (Stata Corporation, College Station, TX, USA).

\section{Aspectos éticos}

El estudio fue aprobado por el Comité Institucional de Ética de la Universidad Peruana Cayetano Heredia y el Comité de Investigación y Ética Institucional del Hospital I Aurelio Díaz Ufano, EsSalud.

\section{RESULTADOS}

De los 240 registros de pacientes con diagnóstico de DMT2 atendidos durante el 2017, se descartaron 28 registros de pacientes: diabetes no especificada $(n=3)$, diabetes tipo $1(n=1)$, registros con información incompleta $(n=22)$, registros no localizados $(n=1)$ y con afiliación tardía a EsSalud ( $n=1)$; quedando un total de 212 registros para el análisis.

De acuerdo a los registros analizados, el $56,1 \%$ fueron mujeres, y se obtuvo una edad promedio de 67,3 años $( \pm 10,1)$, con instrucción básica (63,4\%) (Tabla 1). Respecto a las condiciones clínicas, los participantes presentaron una mediana del tiempo de enfermedad y de aseguramiento de 8 años [ \pm rango intercuartil 
Tabla 1. Características sociodemográficas y clínicas de pacientes con diagnóstico de diabetes mellitus tipo 2, atendidos en consulta externa del Hospital I Aurelio Diaz Ufano, Lima- Perú, 2017.

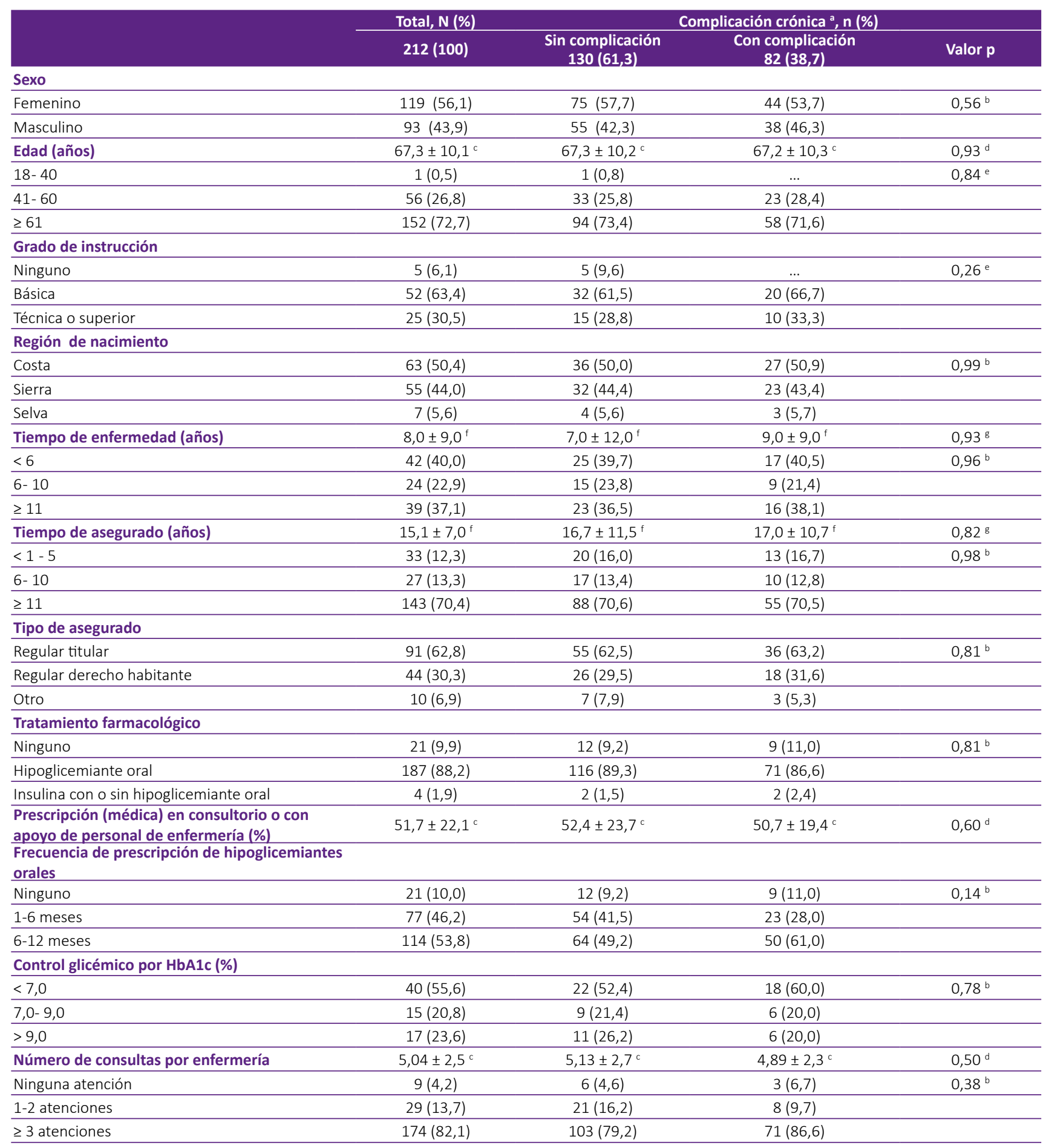

a Población total clasificada en 2 subgrupos: con y sin complicación crónica por DMT2.

b Valor $p$ de la prueba de chi-cuadrado.

c Media \pm desviación estándar.

¿ Valor $\mathrm{p}$ de la prueba de T de Student.

e Valor $p$ de la prueba de exacta de Fisher.

${ }^{f}$ Mediana \pm rango intercuartil.

' Valor $\mathrm{p}$ de la prueba de $\mathrm{U}$ de Mann-Whitney.

Continuación de la tabla 1 
Tabla 1. Características sociodemográficas y clínicas de pacientes con diagnóstico de diabetes mellitus tipo 2, atendidos en consulta externa del Hospital I Aurelio Diaz Ufano, Lima- Perú, 2017.

\begin{tabular}{|c|c|c|c|c|}
\hline & \multirow{2}{*}{$\begin{array}{c}\text { Total, N (\%) } \\
212(100)\end{array}$} & \multicolumn{3}{|c|}{ Complicación crónica a , n (\%) } \\
\hline & & $\begin{array}{c}\text { Sin complicación } \\
130(61,3)\end{array}$ & $\begin{array}{c}\text { Con complicación } \\
82(38,7)\end{array}$ & Valor $p$ \\
\hline \multicolumn{5}{|c|}{ Frecuencia de consultas por medicina interna } \\
\hline Ninguna atención & $153(72,2)$ & $102(78,5)$ & $51(62,2)$ & $0,04^{\mathrm{b}}$ \\
\hline 1-2 atenciones & $55(25,9)$ & $26(20,0)$ & $29(35,4)$ & \\
\hline$\geq 3$ atenciones & $4(1,9)$ & $2(1,5)$ & $2(2,4)$ & \\
\hline \multicolumn{5}{|l|}{ Frecuencia de consultas por oftalmología } \\
\hline Ninguna atención & $167(78,8)$ & $103(79,2)$ & $64(78,0)$ & $0,08^{\mathrm{b}}$ \\
\hline 1-2 atenciones & $33(15,6)$ & $23(17,7)$ & $10(12,2)$ & \\
\hline$\geq 3$ atenciones & $12(5,6)$ & $4(3,1)$ & $8(9,8)$ & \\
\hline \multicolumn{5}{|l|}{ Frecuencia de consultas por urología } \\
\hline Ninguna atención & $178(84,0)$ & $113(86,9)$ & $65(79,3)$ & $0,31^{b}$ \\
\hline 1-2 atenciones & $19(9,0)$ & $9(6,9)$ & $10(12,2)$ & \\
\hline$\geq 3$ atenciones & $15(7,0)$ & $8(6,1)$ & $7(8,5)$ & \\
\hline \multicolumn{5}{|l|}{ Frecuencia de consultas por cardiología } \\
\hline Ninguna atención & $185(87,3)$ & $115(88,5)$ & $70(85,4)$ & $0,56^{b}$ \\
\hline 1-2 atenciones & $22(10,4)$ & $13(10,0)$ & $9(11,0)$ & \\
\hline$\geq 3$ atenciones & $5(2,4)$ & $2(1,5)$ & $3(3,6)$ & \\
\hline \multicolumn{5}{|c|}{ Frecuencia de consultas por medicina familiar } \\
\hline Ninguna atención & $191(90,1)$ & $118(90,8)$ & $73(89,0)$ & $0,89^{b}$ \\
\hline 1-2 atenciones & $19(9,0)$ & $11(8,5)$ & $8(9,8)$ & \\
\hline$\geq 3$ atenciones & $2(0,9)$ & $1(0,8)$ & $1(1,2)$ & \\
\hline Antecedentes de visita a emergencia & $31(14,6)$ & $17(13,1)$ & $14(17,1)$ & $0,42^{b}$ \\
\hline Antecedentes de hospitalización & $1(0,5)$ & $\ldots$ & $1(1,2)$ & $\ldots$ \\
\hline
\end{tabular}

a Población total clasificada en 2 subgrupos: con y sin complicación crónica por DMT2.

b Valor $p$ de la prueba de chi-cuadrado.

c Media \pm desviación estándar.

${ }^{d}$ Valor $p$ de la prueba de T de Student.

e Valor $p$ de la prueba de exacta de Fisher.

${ }^{\mathrm{f}}$ Mediana \pm rango intercuartil.

g Valor $\mathrm{p}$ de la prueba de $\mathrm{U}$ de Mann-Whitney.

(RIC): 9,0] y 15,1 años ( \pm RIC: 7,0), respectivamente. El $23,8 \%$ de los participantes fueron diagnosticados de DMT2 antes de estar asegurado, esto no estuvo asociado a presentar alguna complicación crónica por DMT2. El tratamiento farmacológico fue principalmente con hipoglicemiantes orales $(88,2 \%)$ y solo el $55,6 \%$ de pacientes alcanzó un óptimo control glicémico $(\mathrm{HbA} 1 \mathrm{c}<7 \%)$. La prescripción médica de hipoglicemiantes orales se brindó mensualmente, siendo el $51,7 \%$ en promedio, dispensada con apoyo del personal de enfermería de acuerdo a resultados de control glicémico. No obstante, solo el $53,8 \%$ de los pacientes solicitaron medicamentos con un rango de 6 a 12 meses. Adicionalmente, el número de consultas por enfermería tuvo una media de 5 ( \pm $D E: 2,5)$ y por medicina general fue de 4,1 ( \pm DE: 1,8$)$, mientras que la media de consultas por medicina interna fue de 0,39 ( \pm DE: 0,7) (Tabla 1).

El 38,7\% de los pacientes presentó alguna complicación crónica, el 92,7\% de estos fueron complicaciones microvasculares y el 7,3\% complicaciones macrovasculares. En el grupo con complicación crónica microvascular, la nefropatía $(48,8 \%)$ fue la más frecuente, seguida de neuropatía diabética $(45,1 \%)$, retinopatía $(6,1 \%)$ y pie diabético (2,4\%). Asimismo, en el grupo con complicación crónica macrovascular, la enfermedad cerebro-vascular (4,8\%) se presentó con mayor frecuencia, seguida de enfermedad isquémica $(2,4 \%)$ y enfermedad arterial periférica (1,2\%). Respecto a las comorbilidades evaluadas, la más frecuente fue hipertensión arterial (50,9\%); de estos, el 82,4\% contaba con tratamiento farmacológico. El 23,1\% de los pacientes con complicaciones crónicas, reportó an- tecedentes familiares de DMT2 y el 20,7\% de hipertensión arterial. El 17,9\% presentó sedentarismo y el $16,5 \%$ presentó nutrición inadecuada. El 31,2\% de los pacientes con complicaciones crónicas tuvieron sobrepeso y el $43,7 \%$ obesidad (Tabla 2 ).

En el modelo ajustado, se encontró un $13 \%$ de mayor prevalencia de número de consultas por medicina general en pacientes con complicación crónica por DMT2 ( $R P=1,13$; 95\% IC: 1,04-1,23; $\mathrm{p}<0,01)$. Asimismo, los pacientes con alguna complicación crónica por DMT2 tuvieron un $7 \%$ de mayor prevalencia de número de pruebas de glicemia en ayunas en comparación con los que no tuvieron alguna complicación crónica ( $R P=1,07 ; 95 \%$ IC: 1,00-1,16; $p=0,06$ ) (Tabla 3). Los resultados de las pruebas de laboratorio de los 212 pacientes evaluados, se muestran en la tabla 4. 
Tabla 2. Complicación, comorbilidad y parámetros de control de pacientes con diagnóstico de diabetes mellitus tipo 2, atendidos en consulta externa del Hospital I Aurelio Diaz Ufano, Lima- Perú, 2017.

\begin{tabular}{|c|c|c|c|c|}
\hline & \multirow{2}{*}{$\begin{array}{c}\text { Total, N (\%) } \\
212(100)\end{array}$} & \multicolumn{3}{|c|}{ Complicación crónica a ${ }^{\mathrm{a}}$ n (\%) } \\
\hline & & $\begin{array}{c}\text { Sin complicación } \\
130(61,3)\end{array}$ & $\begin{array}{l}\text { Con complicación } \\
82(38,7)\end{array}$ & Valor pb \\
\hline Complicación crónica microvascular & $76(36,9)$ & NA & $76(92,7)$ & NA \\
\hline Nefropatía diabética & $40(18,9)$ & NA & $40(48,8)$ & NA \\
\hline Neuropatía diabética & $37(17,4)$ & NA & $37(45,1)$ & NA \\
\hline Retinopatía diabética & $5(2,4)$ & NA & $5(6,1)$ & NA \\
\hline Pie diabético & $2(0,9)$ & NA & $2(2,4)$ & NA \\
\hline Complicación crónica macrovascular & $6(4,4)$ & NA & $6(7,3)$ & NA \\
\hline Enfermedad cerebro-vascular & $4(1,9)$ & NA & $4(4,8)$ & NA \\
\hline Enfermedad isquémica & $2(0,9)$ & NA & $2(2,4)$ & NA \\
\hline Enfermedad arterial periférica & $1(0,5)$ & NA & $1(1,2)$ & NA \\
\hline Hipertensión arterial & $108(50,9)$ & $65(50)$ & $43(52,4)$ & 0,73 \\
\hline Hipertensión arterial sin tratamiento & $19(17,6)$ & $11(16,9)$ & $8(18,6)$ & 0,82 \\
\hline Hipertensión arterial con tratamiento & $89(82,4)$ & $54(83,1)$ & $35(81,4)$ & \\
\hline Dislipidemia & $71(33,5)$ & $49(37,7)$ & $22(26,8)$ & 0,10 \\
\hline Anemia & $21(14,8)$ & $11(12,8)$ & $10(17,9)$ & 0,41 \\
\hline Artrosis & $22(10,4)$ & $12(9,2)$ & $10(12,2)$ & 0,49 \\
\hline Infección urinaria & $20(9,4)$ & $14(10,8)$ & $6(7,3)$ & 0,40 \\
\hline Glaucoma & $8(3,8)$ & $4(3,1)$ & $4(4,9)$ & 0,50 \\
\hline Antecedente familiar de diabetes mellitus & $49(23,1)$ & $29(22,3)$ & $20(24,4)$ & 0,72 \\
\hline Antecedente familiar de hipertensión arterial & $44(20,7)$ & $27(20,8)$ & $17(20,7)$ & 0,73 \\
\hline Sedentarismo & $38(17,9)$ & $24(18,5)$ & $14(17,1)$ & 0,80 \\
\hline Nutrición inadecuada & $35(16,5)$ & $21(16,1)$ & $14(17,1)$ & 0,86 \\
\hline \multicolumn{5}{|l|}{ Categorías de índice de masa corporal (kg/m2) } \\
\hline Normopeso $(19,0-24,9)$ & $24(20,7)$ & $12(17,6)$ & $12(25,0)$ & 0,60 \\
\hline Sobrepeso $(25,0-29,9)$ & $40(34,5)$ & $25(36,8)$ & $15(31,2)$ & \\
\hline Obesidad $(\geq 30,0)$ & $52(44,8)$ & $31(45,6)$ & $21(43,7)$ & \\
\hline $\begin{array}{l}\text { Días entre consulta médica y toma de muestra en } \\
\text { laboratorio }\end{array}$ & $40,7 \pm 21,9^{c}$ & $40,7 \pm 24,3^{c}$ & $40,8 \pm 17,4^{c}$ & $0,97^{d}$ \\
\hline Días entre toma de muestra y resultados de laboratorio & $1,2 \pm 2,4^{c}$ & $1,23 \pm 3,0^{c}$ & $1,0 \pm 0,6^{c}$ & $0,57^{d}$ \\
\hline Días entre consulta médica y resultado de laboratorio & $41,7 \pm 21,9^{c}$ & $42,0 \pm 24,6^{c}$ & $41,3 \pm 16,9^{c}$ & $0,84^{d}$ \\
\hline Número de pruebas de glicemia en ayunas & $3,0 \pm 2,2^{c}$ & $2,7 \pm 2,2^{c}$ & $3,3 \pm 2,2^{c}$ & $0,06^{d}$ \\
\hline Glicemia en ayunas $(\mathrm{mg} / \mathrm{dl})^{\mathrm{g}}$ & $155,0 \pm 53,6^{c}$ & $157,6 \pm 56,6^{c}$ & $150,9 \pm 48,3^{c}$ & $0,39^{d}$ \\
\hline $\mathrm{HbA1c}(\%)$ & $7,3 \pm 2,1^{c}$ & $7,5 \pm 2,1^{c}$ & $7,1 \pm 2,2^{c}$ & $0,57^{d}$ \\
\hline Colesterol total $(\mathrm{mg} / \mathrm{dl})$ & $190,9 \pm 37,0^{c}$ & $191,5 \pm 37,2^{c}$ & $190,0 \pm 37,0^{c}$ & $0,80^{d}$ \\
\hline Colesterol LDL (mg/dl) & $104,5 \pm 31,8^{c}$ & $103,8 \pm 32,8^{c}$ & $105,8 \pm 30,1^{c}$ & $0,71^{d}$ \\
\hline Colesterol HDL (mg/dl) & $48,2 \pm 17,6^{c}$ & $49,3 \pm 19,6^{c}$ & $46,5 \pm 13,7^{c}$ & $0,36^{d}$ \\
\hline Triglicéridos (mg/dl) & $179,6 \pm 89,8^{c}$ & $176,8 \pm 93,3^{c}$ & $184,2 \pm 84,6^{c}$ & $0,60^{d}$ \\
\hline Microalbuminuria (mg/24 horas) & $6,0 \pm 17,0^{e}$ & $5,0 \pm 11,0^{e}$ & $8,4 \pm 23,0^{e}$ & $0,25^{f}$ \\
\hline Hemoglobina & $13,6 \pm 1,3^{c}$ & $13,6 \pm 1,24^{c}$ & $13,6 \pm 1,4^{c}$ & $0,88^{d}$ \\
\hline Presión arterial sistólica $(\mathrm{mmHg})$ & $123,3 \pm 14,5^{c}$ & $123,0 \pm 9,0^{c}$ & $123,8 \pm 19,6^{c}$ & $0,77^{d}$ \\
\hline Presión arterial diastólica (mmHg) & $74,3 \pm 5,4^{c}$ & $73,9 \pm 5,6^{c}$ & $74,9 \pm 5,0^{c}$ & $0,30^{d}$ \\
\hline
\end{tabular}

a Población total clasificada en 2 subgrupos: con y sin complicación crónica por DMT2.

${ }^{b}$ Valor $\mathrm{p}$ de la prueba de chi-cuadrado respecto a los grupos con y sin complicación crónica, a menos que se indique lo contrario.

c Media \pm desviación estándar.

${ }^{\mathrm{d}}$ Valor $\mathrm{p}$ de la prueba de T de Student.

e Mediana \pm rango intercuartil.

${ }^{f}$ Valor $\mathrm{p}$ de la prueba de $\mathrm{U}$ de Mann-Whitney.

${ }^{g}$ Glicemia con sangre venosa (prueba estándar de laboratorio) o capilar (hemoglucotest).

NA: No aplica. 
Tabla 3. Análisis bivariado y multivariado de factores asociados a complicación crónica por diabetes mellitus tipo 2 , en pacientes atendidos en consulta externa del Hospital I Aurelio Diaz Ufano, Lima- Perú, 2017

\begin{tabular}{|c|c|c|c|c|c|c|}
\hline & \multicolumn{6}{|c|}{ Complicación crónica ${ }^{a}$} \\
\hline & \multicolumn{3}{|c|}{ Modelo Crudo } & \multicolumn{3}{|c|}{ Modelo Ajustado } \\
\hline & $\mathbf{R P}$ & IC 95\% & Valor $\mathbf{p}^{\mathbf{b}}$ & $\mathbf{R P}$ & IC $95 \%$ & Valor $\boldsymbol{p}^{\mathrm{b}}$ \\
\hline \multicolumn{7}{|l|}{ Sexo } \\
\hline Femenino & Ref & & & Ref & & \\
\hline Masculino & 1,05 & $(0,79-1,55)$ & 0,56 & 1,12 & $(0,79-1,56)$ & 0,53 \\
\hline Edad (años) & 1,00 & $(0,98-1,02)$ & 0,93 & 0,99 & $(0,97-1,01)$ & 0,51 \\
\hline Hipertensión arterial & 1,06 & $(0,75-1,49)$ & 0,73 & 1,08 & $(0,77-1,53)$ & 0,65 \\
\hline Número de pruebas de glicemia en ayunas ${ }^{c}$ & 1,07 & $(1,00-1,15)$ & 0,06 & 1,07 & $(1,00-1,15)$ & 0,08 \\
\hline Número de consultas por medicina general & 1,13 & $(1,04-1,23)$ & $<0,01$ & 1,13 & $(1,04-1,23)$ & $<0,01$ \\
\hline
\end{tabular}

${ }^{a}$ Complicación crónica micro y macrovascular.

${ }^{\text {b }}$ Valor $\mathrm{p}$ del modelo de regresión de poisson. El modelo ajustado incluye a todas las variables presentes en el cuadro.

c Glicemia con sangre venosa (prueba estándar de laboratorio) o capilar (hemoglucotest).

\section{DISCUSIÓN}

Nuestro estudio evaluó factores asociados a complicación crónica micro y macrovascular en pacientes con DMT2 atendidos en un hospital de atención primaria basado en la revisión de registros hospitalarios utilizados en la práctica médica rutinaria. Entre los principales resultados del análisis con múltiples variables, destaca la asociación significativa con el número de consultas por medicina general y consultas por medicina interna. Este hospital general brinda atención primaria, los pacientes con DMT2 reciben consultas por medicina general e interna, y monitoreos mensuales por enfermería (17). De esta forma, cada 3 meses o ante alteraciones de la glicemia, son derivados al médico general o internista para el manejo clínico y farmacológico. El establecimiento con enfoque en atención primaria solo cuenta con acceso a tratamiento de fármacos hipoglucemiantes; por tanto, los médicos generales o internistas derivan a los pacientes a hospitales de mayor complejidad para el tratamiento con insulinoterapia o atenciones de especialidades médicas.

En nuestro estudio, el control glicémico ( $\mathrm{HbA} 1 \mathrm{c}$ menor a $7 \%$ ) fue en el $55,6 \%$ de la población. Esto evidencia que los esfuerzos para el control de la DMT2 en ámbitos de atención primaria aún son insuficientes. Sin embargo, estos resultados corresponden solo al 38,7\% de los 212 registros evaluados, debido a que este examen no se reportó en el resto de las fuentes revisadas. Los resultados de la prueba de $\mathrm{HbA} 1 \mathrm{c}$ son realizados en hospitales externos especializados, remitidos manualmente al centro de origen, con el consecuente reporte en el sistema de salud en un tiempo prolongado. Este hallazgo, es discrepante con la identificación de niveles de HbA1c inferiores a $7 \%$ en el $70 \%$ de población española con edad igual o menor de 75 años ${ }^{(18)}$.

Del total de pacientes evaluados, se identificó un 36,9\% de pacientes con complicación crónica microvascular; principalmente la nefropatía diabética $(18,9 \%)$ y neuropatía diabética $(17,4 \%)$, retinopatía diabética $(2,4 \%)$ y pie diabético $(0,9 \%)$. En el 2011, se identificó un 24,3\% de nefropatía diabética en pacientes atendidos en el Centro de Diabetes e Hipertensión de la Seguridad Social ${ }^{(19)}$. Esta diferencia podría deberse a que el hospital evaluado cuenta con un programa de cuidado integral de hipertensión arterial en adultos y brinda pruebas de laboratorio para perfil renal ${ }^{(20)}$. En la actualidad, hospitales de EsSalud como Díaz Ufano, Voto Bernales, Emergencias Grau, II Ramón Castilla y II Vi- tarte se benefician del programa preventivo renal en EsSalud que brinda evaluación a pacientes en riesgo y con complicaciones renales ${ }^{(21)}$.

Se identificó $17,4 \%$ de neuropatía periférica en pacientes con DMT2; sin embargo, otros estudios identificaron un $96,8 \%$ de neuropatía periférica en pacientes con DMT2 según el análisis de velocidad de conducción nerviosa y 45\% según el instrumento de evaluación de neuropatía de Michigan ${ }^{222}$. Además, identificamos 2,4\% de retinopatía diabética; algunos estudios han identificado una prevalencia de $21,2 \%$ de retinopatía diabética en centros de atención primaria ${ }^{(23)}$. En nuestro estudio, el diagnóstico de retinopatía diabética pudiera estar subreportado debido a que para su confirmación los pacientes son derivados a hospitales de mayor complejidad que cuentan con profesionales y equipos para su detección y manejo. Así, un estudio en el hospital Almenara de EsSalud en Lima identificó prevalencia de 60,9\% de retinopatía diabética ${ }^{(24,25)}$.

Ante el subregistro clínico, es relevante contar con sistemas de información interconectados entre los establecimientos de una sistema de salud para mejorar el manejo clínico de pacientes ${ }^{(26)}$. En este contexto, EsSalud ha implementado un 
Tabla 4. Resultados de pruebas de laboratorio de pacientes con diagnóstico de diabetes mellitus tipo 2, atendidos en consulta externa del Hospital I Aurelio Diaz Ufano, Lima- Perú, 2017.

\begin{tabular}{|c|c|c|c|c|}
\hline & \multirow{2}{*}{$\begin{array}{c}\text { Total, N (\%) } \\
212(100)\end{array}$} & \multicolumn{2}{|c|}{ Complicación crónicaa, n (\%) } & \multirow[b]{2}{*}{ Valor $p^{b}$} \\
\hline & & $\begin{array}{l}\text { Sin complicación } \\
130(61,3)\end{array}$ & $\begin{array}{c}\text { Con complicación } \\
82(38,7)\end{array}$ & \\
\hline \multicolumn{5}{|c|}{ Glicemia en ayunas (mg/dl) } \\
\hline$<70$ & $1(0,5)$ & $0(0,0)$ & $1(1,3)$ & 0,41 \\
\hline $70-100$ & $15(7,6)$ & $10(8,2)$ & $5(6,6)$ & \\
\hline$>100$ & $182(91,9)$ & $112(91,8)$ & $70(92,1)$ & \\
\hline \multicolumn{5}{|c|}{ Control glicémico por HbA1c (\%) } \\
\hline$<7,0$ & $40(55,6)$ & $22(52,4)$ & $18(60,0)$ & 0,78 \\
\hline $7,0-9,0$ & $15(20,8)$ & $9(21,4)$ & $6(20,0)$ & \\
\hline$>9,0$ & $17(23,6)$ & $11(26,2)$ & $6(20,0)$ & \\
\hline \multicolumn{5}{|c|}{ Colesterol total (mg/dl) } \\
\hline$<200$ & $106(60,2)$ & $65(60,2)$ & $41(60,3)$ & 0,99 \\
\hline$\geq 200$ & $70(39,8)$ & $43(39,8)$ & $27(39,7)$ & \\
\hline \multicolumn{5}{|c|}{ Colesterol LDL (mg/dl) } \\
\hline$<100$ & $59(40,4)$ & $38(41,3)$ & $21(38,9)$ & 0,77 \\
\hline$\geq 100$ & $87(59,6)$ & $54(58,7)$ & $33(61,1)$ & \\
\hline \multicolumn{5}{|c|}{$\begin{array}{l}\text { Colesterol HDL en individuos de sexo femenino } \\
(\mathrm{mg} / \mathrm{dl})(\mathrm{n}=83)\end{array}$} \\
\hline$\leq 50$ & $50(60,2)$ & $30(56,6)$ & $20(66,7)$ & 0,25 \\
\hline$>50$ & $33(39,8)$ & $23(43,4)$ & $10(33,3)$ & \\
\hline \multicolumn{5}{|c|}{$\begin{array}{l}\text { Colesterol HDL en individuos de sexo masculino } \\
(\mathrm{mg} / \mathrm{dl})(\mathrm{n}=61)\end{array}$} \\
\hline$\leq 40$ & $25(41,0)$ & $17(45,9)$ & $8(33,3)$ & 0,24 \\
\hline$>40$ & $36(59,0)$ & $20(54,1)$ & $16(66,7)$ & \\
\hline \multicolumn{5}{|c|}{ Triglicéridos (mg/dl) } \\
\hline$<150$ & $78(45,3)$ & $48(45,7)$ & $30(44,8)$ & 0,90 \\
\hline$\geq 150$ & $94(54,6)$ & $57(54,3)$ & $37(55,2)$ & \\
\hline \multicolumn{5}{|c|}{ Microalbuminuria (mg/24 horas) } \\
\hline$\leq 30$ & $78(82,1)$ & $45(83,3)$ & $33(80,5)$ & 0,72 \\
\hline$>30$ & $17(17,9)$ & $9(16,7)$ & $8(19,5)$ & \\
\hline \multicolumn{5}{|c|}{ Proteinuria (g/24 horas) } \\
\hline$\leq 150$ & $11(84,6)$ & $5(83,3)$ & $6(85,7)$ & 0,91 \\
\hline$>150$ & $2(15,4)$ & $1(16,7)$ & $1(14,3)$ & \\
\hline \multicolumn{5}{|c|}{ Presión arterial sistólica (mmHg) } \\
\hline$\leq 130$ & $99(83,9)$ & $60(88,2)$ & $39(78,0)$ & 0,13 \\
\hline$>130$ & $19(16,1)$ & $8(11,8)$ & $11(22,0)$ & \\
\hline \multicolumn{5}{|c|}{ Presión arterial diastólica ( $\mathrm{mmHg}$ ) } \\
\hline$\leq 80$ & $109(94,0)$ & $64(95,5)$ & $45(91,8)$ & 0,41 \\
\hline$>80$ & $7(6,0)$ & $3(5,5)$ & $4(8,2)$ & \\
\hline
\end{tabular}

a Población total clasificada en 2 subgrupos: con y sin complicación crónica por DMT2.

${ }^{b}$ Valor $p$ de la prueba de chi-cuadrado respecto a los grupos con y sin complicación crónica, a menos que se indique lo contrario Cada parámetro de laboratorio difiere en el " $n$ " 
sistema de historia clínica informatizada para receta médica, citas, farmacia, laboratorio y el sistema de aseguramiento ${ }^{(27)}$; sin embargo, se requiere ampliar el registro de fichas de pacientes crónicos con pluripatologías, ya que son pacientes que requieren atenderse en el nivel primario y áreas especializadas. Contar con un sistema integrado entre establecimientos mejoraría la calidad de información y garantizaría una fuente de investigación para la mejora de la salud de los pacientes.

Nuestros hallazgos deben entenderse bajo las siguientes limitaciones. Primero, el subregistro de información en los registros clínicos podría limitar la calidad de la información. Segundo, el inadecuado registro de los diagnósticos CIE podría añadir un sesgo de mala clasificación; para ello, corroboramos la información con diversos registros clínicos para asegurar la correcta clasificación del diagnóstico CIE de los pacientes y sus complicaciones. Y tercero, el uso de fuentes secundarias de información.

En conclusión, el 38,7\% de los pacientes evaluados ( $n=212$ ) tuvieron alguna complicación crónica, principalmente nefropatía $(48,8 \%)$ y neuropatía $(45,1 \%)$. La hipertensión arterial $(52,4 \%)$ y la obesidad $(43,7 \%)$ fueron las comorbilidades más frecuentes en pacientes con complicaciones crónicas. Las complicaciones por DMT2 estuvieron asociadas al número de consultas médicas y monitoreo glicémico. La complicación por DMT2 estuvo asociada a mayor número de consultas médicas y monitoreo glicémico, probablemente como respuesta tardía a los efectos de la enfermedad. La prevalencia de sobrepeso y obesidad fue elevada en estos pacientes. Es fundamental una mejora en la calidad informativa de las historias clínicas y los registros de seguimiento por enfermería, dado que son fuente valiosa para la generación de evidencias.

\section{REFERENCIAS BIBLIOGRÁFICAS}

1. Punthakee Z, Goldenberg R, Katz P. Definition, Classification and Diagnosis of Diabetes, Predia- betes and Metabolic Syndrome. Canadian journal of diabetes. 2018;42 Suppl 1:S10-s15. DOI: https:// doi.org/10.1016/j.jcjd.2017.10.003

2. Association AD. Classification and Diagnosis of Diabetes: Standards of Medical Care in Diabetes-2019. Diabetes Care. 2019;42(Supplement 1):S13-28. DOI: https://doi.org/10.2337/dc19-S002

3. International Diabetes Federation. IDF Diabetes Atlas Eighth Edition 2017 [Internet]. International Diabetes Federation, 2017. 150 p. Disponible en http://www.diabetesatlas.org/

4. Cho NH, Shaw JE, Karuranga S, Huang Y, da Rocha Fernandes JD, Ohlrogge AW, et al. IDF Diabetes Atlas: Global estimates of diabetes prevalence for 2017 and projections for 2045. Diabetes Res Clin Pract. 2018;138:271-81. DOI: 10.1016/j.diabres.2018.02.023

5. Carbone S, Del Buono MG, Ozemek C, Lavie CJ. Obesity, risk of diabetes and role of physical activity, exercise training and cardiorespiratory fitness. Progress in cardiovascular diseases. 2019; 62(4): 327-333. DOI: 10.1016/j.pcad.2019.08.004

6. Instituto Nacional de Estadistica e Informática. Perú Enfermedades No Transmisibles y Transmisibles, 2018. Disponible en: https://www.inei.gob.pe/ media/MenuRecursivo/publicaciones_digitales/Est/ Lib1657/index1.html

7. Luppens D, Piette C, Radermecker RP, Scantamburlo G, Ansseau M, Pitchot W. Depression and type 2 diabetes: etiopathogenic analysis of a frequent comorbidity. Rev Med Liege. 2014;69(11):611-7.

8. Riederer P, Korczyn AD, Ali SS, Bajenaru O Choi MS, Chopp M, et al. The diabetic brain and cognition. Journal of neural transmission. 2017;124(11):1431-54. DOI: 10.1007/s00702-0171763-2

9. Baker MA, Harries AD, Jeon CY, Hart JE, Kapur $A$, Lonnroth $\mathrm{K}$, et al. The impact of diabetes on tuberculosis treatment outcomes: a systematic review. BMC medicine. 2011;9:81. DOI: https://doi. org/10.1186/1741-7015-9-81

10. Lastra G, Syed S, Kurukulasuriya LR, Manrique C, Sowers JR. Type 2 diabetes mellitus and hypertension: an update. Endocrinol Metab Clin North Am. 2014;43(1):103-22. DOI: 10.1016/j.ecl.2013.09.005

11. van Wijngaarden RPT, Overbeek JA, Heintjes EM Schubert A, Diels J, Straatman H, et al. Relation Between Different Measures of Glycemic Exposure and Microvascular and Macrovascular Complications in Patients with Type 2 Diabetes Mellitus: An Observational Cohort Study. Diabetes therapy: research, treatment and education of diabetes and related disorders. 2017;8(5):1097-109. DOI: 10.1007/s13300-017-0301-4

12. Seuring T, Archangelidi O, Suhrcke M. The Economic Costs of Type 2 Diabetes: A Global Systematic Review. Pharmacoeconomics. 2015;33(8):811-31. DOI: 10.1007/s40273-015-0268-9

13. Arredondo A, Azar A, Recaman AL. Diabetes, a global public health challenge with a high epidemiological and economic burden on health systems in Latin America. Global public health. 2018;13(7):7807. DOI: 10.1080/17441692.2017.1316414

14. Carrillo-Larco RM, Barengo NC, Albitres-Flores L, Bernabe-Ortiz A. The risk of mortality among people with type 2 diabetes in Latin America: A systematic review and meta-analysis of population-based cohort studies. Diabetes/metabolism research and reviews. 2019;35(4):e3139. DOI: 10.1002/dmrr.3139

15. Rao Kondapally Seshasai S, Kaptoge S, Thompson A, Di Angelantonio E, Gao P, Sarwar N, et al. Diabetes mellitus, fasting glucose, and risk of causespecific death. N Engl J Med. 2011;364(9):829-41. DOI: 10.1056/NEJMoa1008862

16. Carrillo-Larco RM, Bernabé-Ortiz A. Diabetes mellitus tipo 2 en Perú: una revisión sistemática sobre la prevalencia e incidencia en población general. Revista Peruana de Medicina Experimental y Salud Publica. 2019;36(1):26-36. DOI: 10.17843/ rpmesp.2019.361.4027

17. EsSalud. Hospital Nivel I Aurelio Díaz Ufano y Peral. Disponible en: http://www.essalud.gob.pe/nuestrasredes-asistenciales/san-juan-de-lurigancho/

18. Asunción Vélez-Escalante BJ-F. Evaluación de los objetivos del triple control alcanzados por pacientes con diabetes mellitus tipo 2 atendidos en las consultas de atención primaria de una zona básica de salud. Medicina General y de Familia. 2016;5(2):458. DOI: https://doi.org/10.1016/j.mgyf.2015.12.008

19. Herrera-Añazco $P$, Taype-Rondan A, Lazo-Porras M, Alberto Quintanilla E, Ortiz-Soriano VM, Hernandez AV. Prevalence of chronic kidney disease in Peruvian primary care setting. BMC nephrology. 2017;18(1):246. DOI: 10.1186/s12882-017-0655-X

20. EsSalud. Cuidado Integral del Paciente con Hipertensión Arterial en el Seguro Social de Salud. Lima, Perú, 2016.

21. Diario Expreso [Internet]. Inician programa preventivo renal en EsSalud, 2018. Disponible en: https:// www.expreso.com.pe/medicina/inician-programapreventivo-renal-en-essalud/

22. Ticse R, Pimentel R, Mazzeti P, Villena J. Elevada frecuencia de neuropatía periférica en pacientes con Diabetes mellitus tipo 2 de un hospital general de Lima-Perú. Revista Medica Herediana. 2013;24:114.

23. Larsen MB, Henriksen JE, Grauslund J, Peto T. Prevalence and risk factors for diabetic retinopathy in 17152 patients from the island of Funen, Denmark. Acta Ophthalmol. 2017;95(8):778-86. DOI: 10.1111/ aos.13449

24. Vallenas Pedemonte F. Prevalencia, diagnóstico y tratamiento de retinopatía diabética en pacientes del servicio de oftalmologia Hospital Nacional Guillermo Almenara Irigoyen de febrero a noviembre 2017 Lima-Perú. Universidad Privada San Juan Bautista; 2018.

25. Cardenas MK, Mirelman AJ, Galvin CJ, Lazo-Porras M, Pinto M, Miranda JJ, et al. The cost of illness attributable to diabetic foot and cost-effectiveness of secondary prevention in Peru. BMC Health Serv Res. 2015;15:483. DOI: https://doi.org/10.1186/ s12913-015-1141-4

26. Pedrera-Carbonell V, Gil-Guillén V, Orozco-Beltrán D, Prieto Erades I, Schwarz-Chavarri G, MoyaGarcia MI. Validez de la historia clínica y sistemas de información en los estudios de actividad asistencial en atención primaria. Atención Primaria. 2005;36(10):550-7. DOI: https://doi.org/10.1016/ S0212-6567(05)70565-5

27. Seguro Social de Salud EsSalud [Internet]. EsSalud implementará historia clínica informatizada en centros de atención primaria, 2016. Disponible en: http://www. essalud.gob.pe/essalud-implementara-historia-clinica-informatizada-en-centros-de-atencion-primaria/ 